\title{
Cannabis-Gesetz in Deutschland - ein Erfolg?
}

Nicole Armbrüster, Britta Ginnow, RA Sebastian Schütze

Bundesverband der Pharmazeutischen Industrie e. V., Berlin

\section{ZUSAMMENFASSUNG}

Seit dem 10. März 2017 ist es erlaubt, Patienten mit schwerwiegenden Erkrankungen nach entsprechender Indikationsstellung und bei fehlender Therapiealternative adäquat mit Cannabisarzneimitteln zu versorgen. Das Gesetz zur „Ände- rung betäubungsmittelrechtlicher und anderer Vorschriften“ („Cannabis-Gesetz“) ermöglicht die Verkehrs- und Verschreibungsfähigkeit von Arzneimitteln auf Basis von Cannabisblüten und -extrakten in standardisierter pharmazeutischer Qualität zu medizinischen Zwecken neben Fertigarzneimitteln. Eine Bestandsaufnahme mit Ausblick.

\section{Hintergrund des Gesetzes}

Bis zum Inkrafttreten des Cannabis-Gesetzes war die Versorgung vor allem von Schmerzpatienten unzureichend. Zugelassene Arzneimittel mit Zubereitungen aus Cannabisblüten oder daraus isolierten Stoffen standen nur sehr begrenzt zur Verfügung. Seit 2011 kann ein Mundspray mit Cannabisextrakt $\left(\right.$ Sativex $^{\circledR}$ ) als Fertigarzneimittel für die Indikation der mittelschweren bis schweren Spastik bei Multipler Sklerose zu Lasten der gesetzlichen Krankenversicherungen (GKV) verordnet werden, wenn andere antispastische Arzneimitteltherapien nicht angemessen gewirkt haben. Daneben ist Canemes ${ }^{\circledR}$ mit dem chemisch definierten Wirkstoff Nabilon seit 2016 in Deutschland zur Behandlung von chemotherapiebedingter Übelkeit und Erbrechen zugelassen, wenn Patienten auf andere Medikamente nicht adäquat ansprechen. Zudem war im Einzelfall mit Bezug auf §73 Abs. 3 AMG der Import Dronabinol-haltiger Arzneimittel, die im Ausland z. B. unter dem Namen Marinol ${ }^{\circledR}$ zugelassen sind, möglich, ebenso eine Anwendung von Dronabinol als Rezepturarzneimittel.

Für den Erwerb von Cannabis (getrocknete Cannabisblüten und (annabisextrakte) zur medizinischen Anwendung aus der Apotheke zum Zweck der ärztlich begleiteten Selbsttherapie war stets eine Ausnahmeerlaubnis des Bundesinstituts für Arzneimittel und Medizinprodukte (BfArM) nach $\S 3$ Abs. 2 BtMG (Betäubungsmittelgesetz) notwendig. Hierbei musste das BfArM im Rahmen einer qualifizierten Plausibilitätsprüfung zu dem Ergebnis kommen können, dass eine Behandlung mit Cannabis mangels geeigneter und verfügbarer Therapiealternativen erforderlich ist und es Hinweise auf die Wirksamkeit von Cannabis bei der vorliegenden Krankheit oder Symptomatik gibt. Für immer mehr schwerkranke Patienten, für die keine verfügbare Therapiealternative vorhanden war, erwies sich die Behandlung mit Cannabis als Chance. Knapp 1000 Patienten erhielten letztlich eine Ausnahmeerlaubnis. Nur in den wenigsten Fällen wurden aber die Therapiekosten von den Krankenversicherungen erstattet, was eine erhebliche Kostenlast für die betroffenen Patienten bedeuten konnte.

Insgesamt handelte es sich bei der Erteilung einer Ausnahmeerlaubnis um ein sehr aufwendiges Verfahren, das sich im Sinne einer adäquaten Patientenversorgung für die breite Anwendung als ungeeignet erwies und keine Dauerlösung darstellte. Die Bundesregierung beschloss daher zu handeln und die Möglichkeit von Cannabis zur medizinischen Anwendung für schwerkranke Patienten zu erweitern und zu erleichtern. Dass dies unter dringendem Handlungsdruck geschah, auch im Zusammenhang mit der Legalisierungsdebatte, spiegelt die Tatsache wider, dass hier erstmalig die Erstattungsfähigkeit nicht allein auf zugelassene Arzneimittel mit entsprechender formalisierter Prüfung auf Wirksamkeit, Sicherheit und pharmazeutischer Qualität beschränkt bleiben sollte, sondern dass die überragenden Ergebnisse in der Therapie den Ausschlag zugunsten der betroffenen Patienten gaben.

Am 19.01.2017 hat der Deutsche Bundestag das Gesetz zur Änderung betäubungsmittelrechtlicher und anderer Vorschriften („Cannabis-Gesetz“) einstimmig beschlossen, das die Verkehrs- und Verschreibungsfähigkeit von Arzneimitteln auf Basis von Cannabisblüten und -extrakten in standardisierter pharmazeutischer Qualität zu medizinischen Zwecken ebenso wie die von entsprechenden Fertigarzneimitteln ermöglicht ( Abb. 1). Seit dem 10.03.2017 ist das Gesetz in Kraft und regelt den Einsatz dieser Arzneimittel als Therapiealternative bei Patienten mit schwerwiegenden Erkrankungen. 


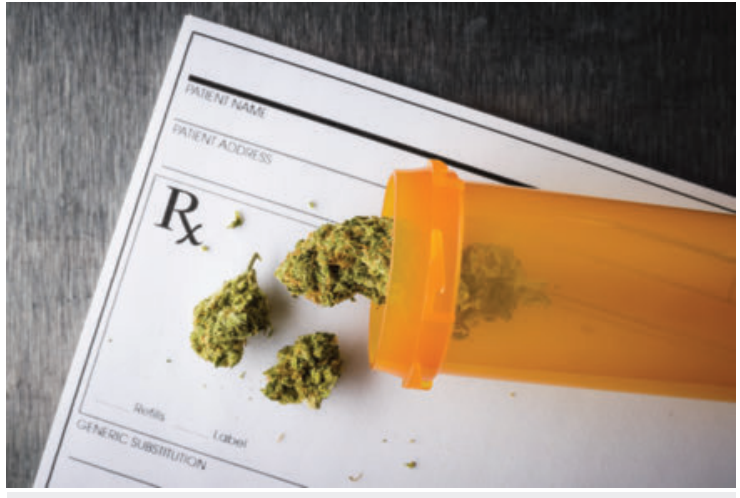

-Abb. 1 Medizinisches Cannabis: verschreibungsfähig mit Hürden in der ärztlichen Praxis. Foto: Adobe Stock / goodmanphoto

\section{Inhalte des Gesetzes}

Das Gesetz soll Ärzten, unabhängig von ihrer Fachrichtung, Sicherheit bei der Therapie und Verschreibung von Cannabis geben, die Erstattung der Kosten für die Therapie regeln und den Anbau von Cannabis zu medizinischen Zwecken in Deutschland möglich machen [1]. Die wichtigsten hierfür notwendigen Änderungen waren:

\section{Betäubungsmittelgesetz}

- „Cannabis, d. h. Marihuana, Pflanzen und Pflanzenteile der zur Gattung Cannabis gehörenden Pflanzen“ wurde von Anlage I in Anlage III des Betäubungsmittelgesetzes überführt und ist damit verkehrs- und verschreibungsfähig.

- Einrichtung einer „Cannabis-Agentur“ zur Steuerung und Kontrolle des Anbaus von Cannabis für medizinische Zwecke in Deutschland. Sie ist ein Fachgebiet in der Abteilung Zulassung 4 im BfArM. Ihr obliegt die Kontrolle von Anbau, Ernte, Verarbeitung, Qualitätsprüfung, Lagerung und Verpackung sowie die Abgabe an Großhändler und Apotheker oder Hersteller. Bis ausreichend Cannabis für medizinische Zwecke in Deutschland angebaut werden kann, wird der Bedarf weiterhin über Importe gedeckt.

\section{Fünftes Buch Sozialgesetzbuch}

$\S 31$ Abs. 6 SGB V wurde eingefügt:

(6) Versicherte mit einer schwerwiegenden Erkrankung haben Anspruch auf Versorgung mit Cannabis in Form von getrockneten Blüten oder Extrakten in standardisierter Qualität und auf Versorgung mit Arzneimitteln mit den Wirkstoffen Dronabinol oder Nabilon, wenn

1. eine allgemein anerkannte, dem medizinischen Standard entsprechende Leistung

a. nicht zur Verfügung steht oder

b. im Einzelfall nach der begründeten Einschätzung der behandelnden Vertragsärztin oder des behandelnden Vertragsarztes unter Abwägung der zu erwartenden Nebenwirkungen und unter

Berücksichtigung des Krankheitszustandes der oder des Versicherten nicht zur Anwendung kommen kann,

2. eine nicht ganz entfernt liegende Aussicht auf eine spürbare positive Einwirkung auf den Krankheitsverlauf oder auf schwerwiegende Symptome besteht. [...]

Gleichzeitig wurde das BfArM mit einer 5 Jahre laufenden nicht-interventionellen Begleiterhebung beauftragt, deren Vorgaben in der Cannabis-Begleiterhebungs-Verordnung (CanBV) festgelegt wurden [2]. Im Rahmen dieser Erhebung sollen Daten zur therapeutischen Anwendung von Cannabisarzneimitteln gesammelt werden.

\section{Erfolge}

Das Cannabis-Gesetz zeigt im Versorgungsalltag Wirkung, immer mehr Patienten profitieren von der Behandlung mit Medizinalcannabis [3]. Laut einer aktuellen Statistik des GKV-Spitzenverbandes beliefen sich die Verordnungen cannabinoidhaltiger Fertigarzneimittel und Zubereitungen von Januar bis September 2018 auf insgesamt 128001 [4]. Die meisten Verordnungen entfielen auf unverarbeitete Cannabisblüten (48 428), gefolgt von cannabishaltigen Zubereitungen (43087) und dem Fertigarzneimittel Sativex ${ }^{\circledR}$ (33 555) ( Tab. 1). Da in der GKV-Statistik auch Folgeverordnungen mitzählen, ist kein direkter Rückschluss auf die genaue Patientenzahl möglich. Bereits im ersten Jahr nach dem Inkrafttreten des Gesetzes wurden damit 12000 bis 14000 Patienten aus medizinischen Gründen mit Cannabis versorgt, wie aus einer parlamentarischen Anfrage im Jahr 2018 hervorgeht [5].

Am häufigsten wurde Medizinalcannabis von Fachärzten für Allgemeinmedizin, hausärztlichen Internisten und Praktischen Ärzten verordnet, die in der Regel chronisch und bereits langjährig erkrankte Patienten betreuen. Daneben stellten Ärzte aus dem neurologischen und psychiatrischen Fachgebiet die häufigsten Cannabisverordnungen aus [3].

\section{Indikationen}

Die meisten genehmigten Anträge auf Erstattung einer Cannabistherapie bezogen sich auf die Indikation Schmerz, gefolgt von Tumorleiden, sonstige neurologische Krankheiten und spezialisierte ambulante Palliativversorgung (SAPV) [6].

\section{Umsetzungsprobleme}

Die Implementierung des Cannabis-Gesetzes verläuft nicht ohne Probleme. Einige Hürden, die sich in der alltäglichen Praxis widerspiegeln, werden im Folgenden dargestellt. 
Tab. 1 Bruttoumsätze und Verordnungen von cannabinoidhaltigen Fertigarzneimitteln und Zubereitungen von Januar bis September 2018 [4].

\begin{tabular}{|l|l|l|}
\hline Arzneimittel & Bruttoumsatz in $€$ & Verordnungen \\
\hline Abrechnung von Cannabis-haltigen Zubereitungen & 17273208 & 43087 \\
\hline Abrechnung von unverarbeiteten Cannabis-Blüten & 22414534 & 48428 \\
\hline $\begin{array}{l}\text { Abrechnung von Cannabis-haltigen Fertigarzneimitteln ohne } \\
\text { Pharmazentralnummer }\end{array}$ & 274584 & 2096 \\
\hline Canemes $^{\circledR}$ & 399740 & 835 \\
\hline Sativex & & 33555 \\
\hline Summe & 10423525 & $\mathbf{1 2 8 0 0 1}$ \\
\hline
\end{tabular}

\section{Genehmigungspraxis der Krankenkassen}

Das bürokratische Erstattungsverfahren erschwert die Nutzung der neuen Therapieoption. Die Anträge werden überwiegend vom Medizinischen Dienst der Krankenversicherung (MDK) geprüft. Die Genehmigungspraxis gestaltet sich regional noch immer sehr unterschiedlich. Die Genehmigungsraten der MDKs variieren je nach Bundesland stark und liegen zwischen 40 und $74 \%$. Die Ablehnungsgründe sind für Ärzte und Patienten oft nicht nachvollziehbar (z. B. formale Ablehnungsgründe der Antragsstellung, fehlende Evidenz, Definition von „schwerwiegender Erkrankung“, Ungeeignetheit potenzieller Alternativtherapien etc.). Weiterhin gab es Fälle, in denen die Krankenkassen die Kostenübernahme zeitlich befristeten oder eine Folgeverordnung ein zweites Mal beantragt werden musste, was das Gesetz so nicht vorgesehen hat und was aus Sicht einer verlässlichen Versorgung von schwerkranken Patienten auch nicht sinnvoll ist. Neben der regionalen Abweichung der Antragsgenehmigungen gibt es auch zwischen den einzelnen Krankenkassen mitunter nennenswerte Abweichungen. Die zeitliche Befristung wurde jedoch bereits durch ein Urteil des Sozialgerichtes Hildesheim vom 21.11.2017 (Aktenzeichen S32 KR 4041/17ER) für unzulässig erklärt. Auch das Bundesversicherungsamt kritisierte diese Praxis [7].

\section{Hohe Kosten}

Für die Apothekenabgabepreise sind bislang die in der Arzneimittelpreisverordnung (AMPreisV) vorgesehenen Apothekerzuschläge auf den Apothekeneinkaufspreis und darüber hinaus die Zuschläge, die die Arbeit der Apotheken abbilden, maßgeblich gewesen. Die Zuschläge auf den Einkaufspreis bei Abgabe von unverarbeiteten Blüten als Stoff beliefen sich bisher auf $100 \%$ (vgl. §4 Abs. 1 AMPreisV), für die Abgabe von Zubereitungen aus Stoffen auf $90 \%$ (vgl. §5 Abs. 1 AMPreisV). Hierdurch wachsen die Ausgaben der Krankenversicherungen, jedoch bei einem vergleichsweise noch sehr moderaten Gesamtkostenfaktor.

\section{Cannabisausschreibung verzögert sich}

Das Cannabis-Gesetz sieht vor, dass der Bedarf an Cannabis langfristig aus deutschem Anbau gedeckt werden soll. Bei der Ausschreibung zum Cannabisanbau sind allerdings gravierende Schwierigkeiten aufgetreten. Das erste Ausschreibungsverfahren scheiterte im März 2018 aufgrund einer Gerichtsentscheidung. Im Rahmen der zweiten Ausschreibung endete die Bewerbungsfrist nach mehrfacher Verschiebung Ende 2018. Die Zuschläge bei der Ausschreibung für mögliche Produzenten von Medizinalcannabis sollen nun erst im zweiten Quartal 2019 bestimmt werden, sodass Cannabis voraussichtlich erst ab 2020 aus deutschem Anbau zur Verfügung steht. Angesichts des wachsenden Bedarfs an Cannabis scheint das geplante Gesamtvolumen von $10400 \mathrm{~kg}$ Cannabis, verteilt auf 4 Jahre mit jeweils $2600 \mathrm{~kg}$, nicht ausreichend bemessen zu sein. Eine Deckung des Bedarfs durch Importe aus dem Ausland dürfte bestehen bleiben. Es wird schon jetzt immer wieder über Lieferschwierigkeiten berichtet.

\section{Geplante Änderungen im GSAV}

Die Erfahrungen mit der Umsetzung des CannabisGesetzes haben gezeigt, dass Nachbesserungsbedarf besteht. Einige notwendige Maßnahmen sollen mit dem Gesetz für mehr Sicherheit in der Arzneimittelversorgung (GSAV) umgesetzt werden (Kabinettsentwurf vom 30.01.2019 [8]):

- Klarstellung, dass eine Anpassung der Dosierung eines Cannabisarzneimittels oder der Wechsel zwischen getrockneten Cannabisblüten oder zwischen Cannabisextrakten in standardisierter Qualität keiner neuen Genehmigung bedarf.

- Nach der Behandlung mit einem Cannabisarzneimittel im Rahmen eines stationären Aufenthaltes unterliegt die vertragsärztliche Verordnung im Anschluss einer verkürzten Genehmigungsfrist von bis zu 3 Tagen nach Antragseingang.

- Apothekenzuschläge für Cannabisarzneimittel (nach $\S 31$ Abs. 6) sollen abweichend vereinbart werden. 
Der Deutsche Apothekerverband (DAV) und der GKV-Spitzenverband (GKV-SV) werden beauftragt, hierfür entsprechende Vereinbarungen zu erarbeiten. Zur Vorbereitung dieser Vereinbarung wird dem GKV-Spitzenverband ein Auskunftsrecht gegenüber Apotheken, pharmazeutischen Unternehmen sowie Arzneimittelgroßhändlern und Parallelimporteuren über vereinbarte Mengen, Preise und Abnehmer eingeräumt. Man geht von einem Einsparvolumen von 25 Mio. Euro aus.

\section{Weitere Vorschläge für Verbesserungen}

Für eine adäquate Versorgung der Patienten gehen die im GSAV geplanten Änderungen nicht weit genug:

\section{Vereinheitlichung der Genehmigungspraxis}

Die Genehmigungspraxis bzw. die Bewertung durch den MDK muss vereinheitlicht werden. Hierfür könnte das BfArM unter Einbindung der betreffenden ärztlichen Fachgesellschaften der Ärzteschaft für die häufigsten Indikationen (z. B. Schmerzen, spezialisierte ambulante Palliativversorgung, Tumorleiden, sonstige neurologische Erkrankungen, Epilepsie, etc.) ein einheitliches und transparentes Prüfungsregime zur Erstattung von medizinischem Cannabis erarbeiten. Darin enthalten sein sollten z. B. die Definition einer schwerwiegenden Erkrankung und ein einheitlicher Bewertungsmaßstab für die Frage der alternativen Therapiemöglichkeiten.

\section{Forschung für Arzneimittel auf Cannabis-Basis fördern}

Die Entwicklung und Zulassung von Cannabinoid-Arzneimitteln in neuen Indikationen ist sehr kostenintensiv. Insbesondere für seltene Indikationen wird es wegen der hohen Entwicklungskosten, des vergleichsweise geringen Umsatzpotenzials und vor allem wegen des AMNOG (Arzneimittelmarktneuordnungsgesetz) wenig wirtschaftliche Anreize geben. Neben den Forschungsaktivitäten der pharmazeutischen Industrie wäre daher ein Commitment des Bundesministeriums für Bildung und Forschung (BMBF) zur Bereitstellung eines Forschungsbudgets für diese Produkte wünschenswert, um die Erkenntnis zu erweitern. Forschungsprojekte könnten auch im Rahmen von Public Private Partnerships (PPP) organisiert werden. 


\section{Fazit}

Cannabis zu medizinischen Zwecken bietet eine große therapeutische Chance bei der Behandlung von verschiedensten Symptomen und Indikationen bei vor allem schwerkranken Patienten. Für eine adäquate Versorgung der Patienten mit Arzneimitteln auf Cannabis-/Cannabinoid-Basis wird von den Krankenversicherungen eine patientenorientiertere Kostenerstattungspolitik gefordert. Die Gesundheitspolitik muss für geeignete Rahmenbedingungen sorgen, um die Forschung und Entwicklung entsprechender Medikamente zu fördern und im Sinne der Patienten einen ungehinderten bzw. unbürokratischen Zugang zu ermöglichen. Insbesondere ist eine Vielfalt der therapeutischen Optionen, bestehend aus Fertig- und Rezepturarzneimitteln unerlässlich, da Patienten auf diese unterschiedlich ansprechen und der individuelle Verlauf der Erkrankungen gerade bei den genannten Indikationen oft sehr verschieden ist. Das Cannabis-Gesetz hat bereits gute Erfolge erzielt. Weitere Regelungen sind aber erforderlich, um für schwerkranke Patienten eine reibungslose Versorgung und kontinuierliche therapeutische Behandlung mit Cannabisarzneimitteln gewährleisten zu können. Das GSAV ist eine Chance, dass der Gesetzgeber hier nachjustiert. Das Gesetz soll am 1. Juli 2019 in Kraft treten.

\section{ABSTRACT}

The German Cannabis legislation for medical purposes: well done or not?

The act of the German parliament that amends the narcotics legislation and other legal provisions allows access for therapeutic application of cannabis medicine for seriously ill patients. It came into force on 10 March 2017 and regulates the eligibility for marketing, prescription and reimbursement of medicines based on cannabis blossoms and extracts of standardized pharmaceutical quality; besides finished medicines. This article presents a review and the future prospects of the German cannabis legislation.

Interessenkonflikt

\section{Korrespondenzadresse}

\section{Dr. Nicole Armbrüster}

Geschäftsfeldleiterin Biologische / Pflanzliche Arzneimittel Bundesverband der Pharmazeutischen Industrie e. V. Friedrichstr. 148

10117 Berlin

E-Mail: narmbruester@bpi.de

\section{Literatur}

[1] Gesetz zur Änderung betäubungsmittelrechtlicher und anderer Vorschriften („Cannabis-Gesetz“), Bundesgesetzblatt Jahrgang 2017 Teil I Nr.11, ausgegeben zu Bonn am 9. März 2017

[2] Cannabis-Begleiterhebungs-Verordnung (CanBV): https:// www.gesetze-im-internet.de/canbv/BJNR052000017.html

[3] Marschall U, L'hoest H, Hennig B: Heilsbringer Cannabis wirklich ein Segen für die Schulmedizin? Auszug aus: BARMER Gesundheitswesen aktuell 2018: 218-271

[4] GKV-Spitzenverband. Sonderbeilage zur GKV-ArzneimittelSchnellinformation für Deutschland: Bruttoumsätze und Verordnungen von Cannabinoidhaltigen Fertigarzneimitteln und Zubereitungen von Januar bis September 2018. https: / / www.gkv-gamsi.de/media/dokumente/gamsi_statistiken/2018/ q3_20/Bundesbericht_GAmSi_201809_konsolidiert_Sonderbei lage_Cannabis.pdf

[5] Bremische Bürgerschaft. Antwort des Senats auf die Kleine Anfrage der Fraktion der FDP vom 18. April 2018. https:// www.bremische-buergerschaft.de/drs_abo/2018-05-29_Drs19-1686_6d584.pdf

[6] Techniker Krankenkasse: Cannabis-Report 2018

[7] Bundesversicherungsamt: Tätigkeitsbericht 2017: 27-28

[8] Entwurf eines Gesetzes für mehr Sicherheit in der Arzneimittelversorgung (GSAV), Kabinettsentwurf vom 30. Januar 2019

Bibliografie

DOI https://doi.org/10.1055/a-0827-8777

Zeitschrift für Phytotherapie 2019; 40: 52-56

(c) Georg Thieme Verlag KG Stuttgart · New York ISSN 0722-348X

Die Autoren sind Mitarbeiter des Bundesverbandes der Pharmazeutischen Industrie, der Interessenvertretung der deutschen Pharmaindustrie in Deutschland und Europa. 